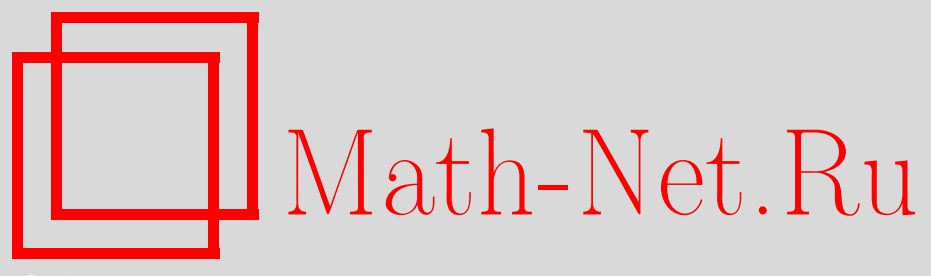

Е. Л. Лакштанов, Коротковолновый предел амплитуды рассеяния в неоднородной среде, УМН, 2007, том 62, выпуск 4, 163-164

DOI: https://doi.org/10.4213/rm7261

Использование Общероссийского математического портала Math-Net.Ru подразумевает, что вы прочитали и согласны с пользовательским соглашением http://www . mathnet.ru/rus/agreement

Параметры загрузки:

IP : 54.147 .182 .235

26 апреля 2023 г., 14:48:48

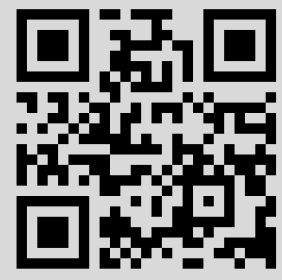




\section{Коротковолновый предел амплитуды рассеяния в неоднородной среде}

\section{Е. Л. Лакштанов}

Рассмотрим задачу рассеяния плоской волны на неоднородностях. Пусть $\Psi(x, k)$ есть единственное решение (см. [1]) уравнения

$$
\left[\Delta_{x}+k^{2} q(x)\right] \Psi(x, k)=0, \quad x=\left(x_{1}, \ldots, x_{n}\right) \in \mathbb{R}^{n},
$$

где $q(x) \in C^{\infty}\left(\mathbb{R}^{n}\right), q(x)>0, q(x) \equiv 1$ при $r=|x|>a, \Psi(x, k)=e^{i k x_{n}}+u(x, k)$ и функция $u(x, k)$ удовлетворяет условию излучения на бесконечности

$$
u(x, k)=f(\omega, k) r^{(1-n) / 2} e^{i k r}\left(1+O\left(r^{-1}\right)\right), \quad r \rightarrow \infty, \quad \omega=\frac{x}{r} \in S^{n-1} .
$$

Функция $f(\omega, k)$ называется амплитудой рассеяния. В этой работе для большого класса потенциалов $q(x) \in C_{0}^{\infty}\left(\mathbb{R}^{n}\right)$ будет получен слабый предел при $k \rightarrow \infty$ меры на сфере $S^{n-1}$ с плотностью $|f(\omega, k)|^{2}$.

Уравнению (1) соответствуют гамильтониан $H=|p|^{2}-q(x), p \in \mathbb{R}^{n}$, и система уравнений Гамильтона

$$
\frac{d x}{d s}=2 p, \quad \frac{d p}{d s}=\nabla q(x) ; \quad x(0)=\left(y^{\prime},-a\right), \quad p(0)=\theta_{0}:=(0, \ldots, 0,1) \in S^{n-1},
$$

где $a>0$ таково, что гиперплоскость $\left\{\left(y^{\prime},-a\right): y^{\prime} \in \mathbb{R}^{n-1}\right\}$ не пересекается с носителем $\operatorname{supp} q(\cdot)$. Предполагается, что система Гамильтона удовлетворяет следующему условию неловушечности.

Условие А. Для любого $c<\infty$ существует такое $T$, что при $s>T$ решение системы (3) лежат в области $|x|>c$.

Обозначим через $\mathscr{I}$ проекцию носителя функции $q(x)-1$ на множество $x_{n}=-a$ :

$$
\mathscr{I}=\left\{\left(y^{\prime},-a\right): \exists a^{\prime}>-a: q\left(y^{\prime}, a^{\prime}\right) \neq 1\right\} \subset \mathbb{R}^{n-1} .
$$

В соответствии с условием неловушечности, каждый луч, выпущенный из точки $\left(y^{\prime},-a\right)$, начиная с некоторого момента двигается по прямой, причем с единичным по модулю вектором скорости (см. [2]), что позволяет определить отображение $J$ : $\mathscr{I} \rightarrow S^{n-1}$.

Заметим, что классическое дифференциальное сечение рассеяния $\left|f_{\mathrm{cl}}\right|^{2}$ системы (3) может быть определено как положительная обобщенная функция (борелевская мера) посредством функционала $\int_{S^{n-1}} \varphi(\omega)\left|f_{\mathrm{cl}}\right|^{2} d \omega=\int_{\mathscr{I}} \varphi(J(y)) d y, \varphi \in C^{\infty}\left(S^{n-1}\right)$. Для того чтобы обобщенная функция $\left|f_{\mathrm{cl}}\right|^{2}$ была регулярной, т. е. соответствующая мера имела плотность, мы предположим выполнение условия невырожденности.

УсловиЕ В. (В.1) Множество критических точек отображения $J$ имеет меру нуль. (В.2) Значение $\theta_{0}$ принимается (за исключением $\partial \mathscr{I}$ ) только в регулярных точках отображения $J$.

Множество критических значений отображения $J$ будем называть множеством $\kappa a-$ устических направлений. Заметим, что по теореме Сарда мера этого множества равна нулю.

Работа выполнена при поддержке Centre for Research on Optimization and Control (CEOC) из "Fundação para a Ciência e a Tecnologia" FCT, и European Community Fund FEDER/POCTI. 
Плотность меры $\left|f_{\mathrm{cl}}\right|^{2}$ можно определить при помощи якобиана отображения $J$. Пусть $\omega_{0}$ - правильное направление (т. е. правильная точка образа), причем прообраз $\omega_{0}$ состоит из $p$ точек $x^{(i)} \in \mathscr{I}, i=1, \ldots, p$. Тогда (см. [2; лемма 1]) существует $p$ окрестностей $\mathscr{M}_{i}=\mathscr{M}_{i}\left(x^{(i)}\right)$ таких, что $J$ диффеоморфно отображает $\mathscr{M}_{i}$ на $\mathscr{L}_{i}=J\left(\mathscr{M}_{i}\right)$.

Обозначим $f_{\mathrm{cl}}^{i}(\omega):=\left|J_{i}(\omega)\right|^{-1 / 2}, \omega \in \mathscr{L}_{i}, i=1, \ldots, p$. Имеем

$$
\int_{\mathscr{L}_{i}}\left|f_{\mathrm{cl}}^{i}(\omega)\right|^{2} d \omega=\int_{\mathscr{M}_{i}} d y^{\prime}=\operatorname{meas}\left(\mathscr{M}_{i}\right), \quad\left|f_{\mathrm{cl}}(\omega)\right|^{2}=\sum_{i=1}^{p}\left|f_{\mathrm{cl}}^{i}(\omega)\right|^{2}, \quad \omega \in \mathscr{L} .
$$

Как следует из (4), функция $f_{\mathrm{cl}} \in L_{2}\left(S^{n-1}\right)$ и $\left\|f_{\mathrm{cl}}\right\|^{2}=\sigma_{\mathrm{cl}}=\operatorname{meas}(\mathscr{I})$, здесь $\sigma_{\mathrm{cl}}-$ так называемое классическое полное сечение рассеяния.

Для формулировки дальнейших условий на функцию $q(\cdot)$ нам потребуются некоторые обозначения для функций, впервые введенных Б. Р. Вайнбергом в [2]. Обозначим через $\Lambda^{n} \subset \mathbb{R}_{x, p}^{2 n}$ лагранжево многообразие, образованное бихарактеристиками (фазовыми кривыми) задачи (3). В качестве (глобальных) координат на $\Lambda^{n}$ можно взять $\left(y^{\prime}, s\right)$, где $y^{\prime} \in \mathbb{R}^{n-1}$; будем называть эти координаты лучевыми. Решая задачу (3), получаем функцию $S=S(x, p) \in C^{\infty}\left(\Lambda^{n}\right): S(x, p)=-a+\int_{L}\langle p, d x\rangle$, где $L-$ отрезок приходящей в точку $(x, p)$ бихарактеристики, заключенный между точкой $(x, p)$ и начальной точкой, для которой $s=0$. Определим функцию $F_{i}(\omega): \mathscr{L}_{i} \rightarrow \mathbb{R}$, $F_{i}(\omega)=S(x, p)-\langle\omega, x\rangle$, где $x \in \mathbb{R}^{n}$ есть произвольная точка луча, выпущенного из $J_{i}^{-1}(\omega)$; это определение не зависит от выбора точки $x$, поскольку $\nabla S(x, p)=\omega$.

В работе [2] показано, что функции $F_{i}(\omega)$ являются фазами семейств лучей, идущих в направлении $\omega$. Мы предположим, что все эти функции существенно различны.

УСловие С. Для любого правильного направления $\omega_{0}$ при всех $1 \leqslant i<j \leqslant p$ множество критических точек функции $F_{i}-F_{j}$, определенной на $\mathscr{L}_{i, j}:=\mathscr{L}_{i} \cap \mathscr{L}_{j}$, имеет меру нуль.

Теорема 1. Пусть потенииал $q(x)$ удовлетворяет условиям А, В и С. Тогда имеет место слабал сходимость

$$
\left|f_{k}\right|^{2} \rightarrow\left|f_{\mathrm{cl}}\right|^{2}+\sigma_{\mathrm{cl}} \delta\left(\theta_{0}\right), \quad k \rightarrow \infty,
$$

где $\left|f_{k}\right|^{2}$ и $\left|f_{\mathrm{cl}}\right|^{2}$ суть мерь на $S^{n-1}$ с плотностями $|f(\omega, k)|^{2} u\left|f_{\mathrm{cl}}(\omega)\right|^{2}$ соответственно.

Эта теорема, в частности, позволяет вычислить асимптотику полного сечения рассеяния и транспортного сечения рассеяния, задаваемых формулами

$$
\sigma=\int_{S^{n-1}}|f(k, \omega)|^{2} d \omega, \quad R=\int_{S^{n-1}}\left(1-\left\langle\omega, \theta_{0}\right\rangle\right)|f(k, \omega)|^{2} d \omega
$$

соответственно. Применяя теорему 1 , мы получаем $\sigma=2 \sigma_{\mathrm{cl}}+o(1), R=R_{\mathrm{cl}}+o(1)$, $k \rightarrow \infty$, где $R_{\mathrm{cl}}$ определяется так же, как и $R$, только при помощи $f_{\mathrm{cl}}(\omega)$.

Теорема 1 в случае рассеяния на препятствии была доказана в [3].

Автор благодарен С. А. Пирогову, который познакомил его с работами Б. Р. Вайнберга.

\section{Список литературы}

[1] А. Н. Тихонов, А. А. Самарский, Уравнения математической физики, Наука, М., 1966. [2] Б. Р. Вайнберг, Функи. анализ и его прилож., 11:4 (1977), 6-18. [3] W. De Roeck, E. L. Lakshtanov, J. Math. Phys., 48:1 (2007), 013501. 